\title{
HAMILTONIAN LOOPS
}

D. A. NORTON

A Hamilton loop is a loop in which every subloop is normal. This definition is somewhat broader than the analogous definition of group theory where a Hamiltonian group is restricted to be non-Abelian. The structure of a Hamiltonian group is well known [4, pp. 129-131 $]^{1}$ -it is the direct product of an Abelian group whose elements have odd order, an Abelian group with exponent 2, and a quaternion group. The purpose of this paper is to investigate the structure of Hamiltonian loops.

It is relatively easy to construct loops of any order (except 4 ) which have no proper subloops and which are therefore trivially Hamiltonian. With a more careful construction it may be shown that given any series of integers $n_{1}, n_{2}, \cdots, n_{k}$ where $n_{i} \neq 4, i=1,2, \cdots, k$, there exists a loop $N_{k}$ of order $n_{1} n_{2} \cdots n_{k}$ with subloops $N_{1}, N_{2}, \cdots$, $N_{k-1}$ of order $\left(n_{1}\right),\left(n_{1} n_{2}\right), \cdots,\left(n_{1} \cdots n_{k-1}\right)$ respectively such that $N_{1} \subset N_{2} \subset \cdots \subset N_{k}$, each loop $N_{i}, i<k$, is normal in $N_{k}$, and $N_{k}$ contains no other proper subloops. ${ }^{2}$ Thus we can construct a Hamiltonian loop with a prescribed series of composition. It becomes apparent that for a complete structure theory some additional hypotheses are necessary.

In Theorem I a necessary and sufficient condition that the direct product of Hamiltonian loops be Hamiltonian is obtained. In Theorems II to VII the structure of a Hamiltonian loop under successively greater restrictions is discussed. In each case where an additional restriction is added to the loop, an example may be constructed to show that there exist loops satisfying all previous conditions but not the additional one, and which do not satisfy the resulting theorem. The simplest assumption yielding significant results is that the loop be power associative.

We shall list a few familiar facts concerning normal subloops. The following lemma is due to Bruck [2, p. 256].

LeMma A. If $x$ and $y$ are given elements of a loop and if two of the three elements $h_{1}, h_{2}, h_{3}$ are given elements of a normal subloop, then the third may be uniquely found as an element of the same subloop where

Presented to the Society, September 1, 1949; received by the editors August 1. 1950 and, in revised form, May 4, 1951.

${ }^{1}$ Numbers in brackets refer to the references cited at the end of the paper.

2 The constructions are cumbersome and since they seem only to have a negativistic value it has not seemed appropriate to include them. 


$$
h_{1} x \cdot h_{2} y=h_{3} \cdot x y \text {. }
$$

As immediate corollaries of this we have $h_{1} x=x h_{4} ; h_{1} x \cdot y=h_{5} \cdot x y$; $x h_{1} \cdot y=x \cdot h_{6} y ; x y \cdot h_{1}=x \cdot y h_{7}$, where, in any equation, if one of $h_{i}$ is given as an element of a normal subloop, the other is uniquely determined as an element of the same subloop. For the following facts, see, for instance, [1]. The order of a normal subloop divides the order of the loop. The order of the union of normal subloops divides the product of their orders. If $A, B$, and $C$ are normal subloops and if $a \in A, b \in B, c \in C$, then $a b=d \cdot b a$ where $d \in A \cap B$; and $a b \cdot c$ $=e(a \cdot b c)$ where $e \in A \cap B \cap C$. If $A \cap B=1$, then the union of $A$ and $B$ is the direct product-that is, $\{A, B\}=A \times B$.

Theorem I. The direct product of two Hamiltonian loops $M$ and $N$ is Hamiltonian if and only if for every set of subloops $H^{\prime} \subset H \subset M$, $K^{\prime} \subset K \subset N$ such that $H / H^{\prime} \cong K / K^{\prime}$, then $H / H^{\prime}$ is in the center of $M / H^{\prime}$ and $K / K^{\prime}$ is in the center of $N / K^{\prime}$.

Proof. If $m, m^{\prime} \in M$ and $n, n^{\prime} \in N$ and if $m m^{\prime}$ indicates multiplication in $M$ and $n n^{\prime}$ indicates multiplication in $N$, then the elements of the direct product $M \times N$ are of the form $(m, n)$ and $\left(m^{\prime}, n^{\prime}\right)$ where multiplication is defined by the law $(m, n)\left(m^{\prime}, n^{\prime}\right)=\left(m m^{\prime}, n n^{\prime}\right)$. Moreover $(m, n)=\left(m^{\prime}, n^{\prime}\right)$ if and only if $m=m^{\prime}$ and $n=n^{\prime}$. If $L$ is a subloop of $M \times N$, then $L$ consists of elements $(h, k)$ where $h \in M$ and $k \in N$. If $1_{m}$ is the unit element of $M$ and $1_{n}$ is the unit element of $N$, then $\left(1_{m}, 1_{n}\right)$ is the unit element of $M \times N$ and any of its subloops. Except where necessary for clarity, we shall omit the subscript and write this as $(1,1)$.

Let $H$ be the set of elements of $M$ and $K$ be the set of elements of $N$ which go to make up the elements of a subloop $L$ of $M \times N$. The set $H$ can be readily seen to be a subloop of $M$ and $K$ a subloop of $N$. Let $H^{\prime}$ be the subset of elements $h^{\prime}$ of $M$ such that $\left(h^{\prime}, 1\right) \in L$, and let $K^{\prime}$ be the subset of elements $k^{\prime}$ of $N$ such that $\left(1, k^{\prime}\right) \in L$. The sets $H^{\prime}$ and $K^{\prime}$ again are easily seen to be loops.

By the Hamiltonian character of $M$ and $N, H^{\prime}$ is a normal subloop of $H$ and $K^{\prime}$ is a normal subloop of $K$. Let $L^{\prime}=H^{\prime} \times K^{\prime}$. Then $L^{\prime}$ is a subloop of $L$. Moreover it is a normal subloop of $M \times N$ and therefore a normal subloop of $L$. Under the homomorphism of $L$ on $L / L^{\prime}$, there is established an isomorphism between the cosets of $H$ in respect to $H^{\prime}$ and the cosets of $K$ in respect to $K^{\prime}$ so that $H / H^{\prime} \cong K / K^{\prime}$.

We have shown that to each subloop $L$ of $M \times N$ there exist two sets of loops, $H^{\prime} \subset H \subset M$ and $K^{\prime} \subset K \subset N$, such that $H / H^{\prime} \cong K / K^{\prime}$. Conversely, given two sets of loops and the indicated isomorphism between the quotient loops, let $H_{1}, H_{2}, \cdots$ be the cosets of $H$ in re- 
spect to $H^{\prime}$ and let $K_{1}, K_{2}, \cdots$ be the cosets of $K$ in respect to $K^{\prime}$, so ordered that $H_{i}$ corresponds to $K_{i}$ under the isomorphism. Then the set of elements

$$
\left(h_{i}, k_{i}\right), \quad h_{i} \in H_{i}, k_{i} \in K_{i}, i=1,2, \cdots,
$$

may be readily seen to make up a loop $L$ which is a subloop of $M \times N$.

The group of permutations generated by all permutations $R_{x} R_{y} R_{x y}^{-1}$ and $R_{y} L_{x} R_{x y}^{-1}$ is the inner mapping group of the loop. Bruck [2, p. 259] has shown that a necessary and sufficient condition that a subloop be normal is that it be mapped into itself by all permutations of the inner mapping group. A central element of the loop is carried into itself by any permutation of the inner mapping group. Let $\mathfrak{T}$ be any mapping of the inner mapping group of the loop $M \times N$; then $\mathfrak{T}=(\mathfrak{U}, \mathfrak{B})$ where $\mathfrak{U}$ and $\mathfrak{B}$ are inner mappings of $M$ and $N$ respective$1 y$, and

$$
(h, k) \mathfrak{T}=(h, k)(\mathfrak{U}, \mathfrak{B})=(h \mathfrak{U}, k \mathfrak{B}) .
$$

If $H / H^{\prime}$ is in the center of $M / H^{\prime}$, each inner mapping of $M / H^{\prime}$ carries each element of $H / H^{\prime}$ into itself; or, that is, each inner mapping $\mathfrak{U}$ of $M$ maps each coset $h H^{\prime}$ of $H$ in respect to $H^{\prime}$ into itself. Similarly if $K / K^{\prime}$ is in the center of $N / K^{\prime}$, each inner mapping $\mathfrak{B}$ of $N$ carries each coset $k K^{\prime}$ of $K$ in respect to $K^{\prime}$ into itself. If $\mathfrak{I}$ is any inner mapping of $M \times N$ and $(h, k)$ is an element of $L$, then $(h, k) \mathfrak{I}$ $=(h, k)(\mathfrak{U}, \mathfrak{B})=(h \mathfrak{u}, k \mathfrak{B})=(\bar{h}, \bar{k})$, where $\bar{h} \in h H^{\prime}$ and $\bar{k} \in k K^{\prime}$. Since the set $\left(h H^{\prime}, k K^{\prime}\right) \subset L$, we have $(\bar{h}, \bar{k}) \in L$ and $L$ is normal.

Conversely, if $L$ is a normal subloop of $M \times N$, and if $\mathfrak{U}$ is any inner mapping of $M$, and $\Im$ the identity transformation of $N$, then $(\mathfrak{U}, \Im)$ is an inner mapping of $M \times N$. If $(h, k) \in L$, then $(h, k)(\mathfrak{U}, \Im)=(h \mathfrak{u}, k)$ and thus $h \mathfrak{U} \in h H^{\prime}$ as proved above. So $\mathfrak{U}$ maps each coset $h H^{\prime}$ into itself and $H / H^{\prime}$ is in the center of $M / H^{\prime}$. Similarly $K / K^{\prime}$ is in the center of $N / K^{\prime}$.

Theorem II. A power associative, Hamiltonian loop $H$ in which every element has finite order is the direct product of p-loops.

Proof. A $p$-loop is a loop in which every element has order a power of the prime number $p$.

Let $H_{i}$ be the set of elements $h \in H$ where the order of $h$ is a power of the prime $p_{i}$. The set $H_{i}$ is a loop. It clearly contains the unit element. If $x, y \in H_{i}$, then there exist integers $r, s$ such that $x^{r}=y^{s}=1$. Consider the element $x y$. By a simple induction proof using Lemma $\mathrm{A}$ we shall show that $(x y)^{r}=x^{r} y^{t}$ for some integer $t$. Suppose $(x y)^{m}=x^{m} y^{n}$ 
for some integer $n$. Then from the power associativity and from Lemma $A$ and its corollaries we have

$$
(x y)^{m+1}=\left(x^{m} y^{n}\right)(x y)=\left(x^{m} x\right) y^{\prime}
$$

for some element $y^{\prime} \in\{y\}$, since $y, y^{n} \in\{y\}$, a normal subloop of $H$. But $y$ has finite order, so that $y^{\prime}=y^{q}$ for some integer $q$, and $(x y)^{m+1}$ $=x^{m+1} y^{q}$. Therefore $(x y)^{r s}=\left(x^{r} y^{t}\right)^{s}=y^{t s}=\left(y^{s}\right)^{t}=1$. But there exist integers $u, v$ such that $r=p_{i}^{u}, s=p_{i}^{0}$, by the definition of $H_{i}$, and $r s=p_{i}^{u+v}$. The element $x y$ belongs to $H_{i}$ and $H_{i}$ is closed under multiplication. If $a x=y$, then $a=y x^{w}$ for some integer $w$, and $a^{r s}=1$ as above, so that $a \in H_{i}$. Similarly, if $x b=y, b \in H_{i}$.

Since $H_{i} \subset H$ and since $H_{i} \cap H_{j}=1, i \neq j$, the product $\prod_{i} H_{i}$ is a direct product. Moreover $\prod_{i} H_{i} \subset H$. Conversely, for any $h \in H,\{h\}$ is a Hamiltonian group and can be written by group theory as the product of $p$-groups so $h=a_{1} a_{2} \cdots$, where $a_{i} \in H_{i}$, and therefore $h \in \prod_{i} H_{i}$. Combining these we have the theorem.

It is possible to give examples of loops which are Hamiltonian and power associative but which do not satisfy the following structure theorems so that some additional restrictions are necessary. Let us assume that the loop is di-associative or, that is, that every two elements of the loop generate a group.

Theorem III. A commutative, di-associative, Hamiltonian loop $H$ is an Abelian group.

Proof. Assume that $H$ is not Abelian. Then there exist elements $a, b, c$ such that

$$
a \cdot b c=d(a b \cdot c), \quad d \neq 1 .
$$

Since $\{a\},\{b\},\{c\}$ are normal subloops of $H, d \in\{a\} \cap\{b\} \cap\{c\}$, and since they are Hamiltonian groups, $d=a^{i}=b^{i}=c^{k}$ for some set of integers $i, j, k$ (not necessarily positive). Let $m=i k j$. Then

$$
(a \cdot b c)^{m}=[d(a b \cdot c)]^{m}=d^{m}(a b \cdot c)^{m} .
$$

Now by the di-associativity and commutativity,

$$
(a \cdot b c)^{m}=a^{m}(b c)^{m}=a^{m} \cdot b^{m} c^{m}=d^{j k} \cdot d^{i k} d^{i j}=d^{i j+i k+j k} .
$$

Similarly $(a b \cdot c)^{m}=d^{i j+i k+j k}$ so, from $(2), d^{m}=1$, and thus $a^{i m}=b^{j m}$ $=c^{k m}=1$. We have shown that every element not in the associator has finite order.

Let $A$ be the subset of $H$ consisting of all elements of finite order. The set $A$ is a loop. It remains to be shown that $A$ is associative. By Theorem II, $A$ is the direct product of $p$-loops, so it will be sufficient 
to show that a commutative, di-associative, Hamiltonian $p$-loop is associative. We use the fact that in an Abelian $p$-group with two generators $a, b$, if the order of $a$ is greater than or equal to the order of $b$, then there exists an element $b_{1}$ such that $\{a, b\}=\left\{a, b_{1}\right\}$ where $\{a\} \cap\left\{b_{1}\right\}=1 .^{3}$

Let $a, b, c$ be three elements of such a $p$-subloop of $H$. Of the orders of the three elements suppose that the order of $a$ is maximal. Since two elements generate a group, there exist elements $b_{1}, c_{1}$ of $H$ such that $\{a, b\}=\left\{a, b_{1}\right\}$ where $\{a\} \cap\left\{b_{1}\right\}=1$, and such that $\{a, c\}$ $=\left\{a, c_{1}\right\}$ where $\{a\} \cap\left\{c_{1}\right\}=1$. Therefore $\left\{a^{r}\right\} \cap\left\{b_{1}^{s}\right\}=1$ for any integers $r, s$ and we have $a^{r} b_{1}^{s} \cdot c^{t}=d\left(a^{r} \cdot b_{1}^{s} c^{t}\right)$ where $d \in\left\{a^{r}\right\} \cap\left\{b_{1}^{s}\right\}$ $\cap\left\{c^{t}\right\}=1$. Then $b=a^{m} b_{1}^{n}$ and $c=a^{p} c_{1}^{q}$ for some set of integers $m, n, p, q$. So $a b \cdot c=\left(a \cdot a^{m} b_{1}^{n}\right) c=\left(a^{m+1} b_{1}^{n}\right) c=a^{m+1}\left(b_{1}^{n} \cdot c\right)=a\left(a^{m} \cdot b_{1}^{n} c\right)$ $=a\left(a^{m} b_{1}^{n} \cdot c\right)=a \cdot b c$. In exactly a similar way it may be shown that the three elements associate for any permutation of the elements or if $b$ or $c$ has the maximal order.

Corollary IIIa. A Hamiltonian, di-associative $p$-loop with $p$ an odd integer is an Abelian group.

Proof. By Theorem III it is sufficient to show that the loop is commutative. If it contains two elements $a, b$ which do not commute, $\{a, b\}$ is a Hamiltonian group and so contains an element of order 4 . Since the loop is Hamiltonian, $p$ must be divisible by 4 ; however, $p$ is assumed odd.

THEOREM IV. In a Hamiltonian, di-associative loop $H$ which is not commutative, every element has finite order.

Proof. If $x$ and $y$ are two elements of $H$ which do not commute, then $\{x, y\}$ is a Hamiltonian group which contains elements $a$ and $b$ which generate a quarternion group so that $a b=b^{3} a, a^{4}=b^{4}=(a b)^{4}$ $=1$, and $a^{2}=b^{2}=(a b)^{2} \neq 1$.

The set of elements of a loop which commute with every element of the loop we shall call the commutator of the loop. If $c$ is not an element of the commutator, there exists an element $z$ of $H$ such that $c z \neq z c$ and $\{c, z\}$ is a Hamiltonian group. Thus $c$ has finite order.

If $c$ is an element of the commutator, and if $a b \cdot c=d(a \cdot b c)$, when squared, this becomes $(a b)^{2} c^{2}=d^{2}(a \cdot b c)^{2}$, which gives

$$
a^{2} c^{2}=d^{2}(a \cdot b c)^{2} \text {. }
$$

Since $d \in\{a\}$, we have $d=1, a, a^{2}$, or $a^{3}$. If $d=a$ or $a^{3},\{a\}=\{d\}$

\footnotetext{
${ }^{3}$ If $a^{m}=b^{n}, a^{s}=b^{t}=1$ are the generating relationships, then $b_{1}=a^{x} b$ where $x=(t-n) m / n^{2}$.
} 
$C\{c\}$ and $\{a, b, c\}=\{b, c\}$, a group, so $d=1$ contrary to our assumption. If we assume $d=1$ or $a^{2}$, then $d^{2}=1$ and (3) becomes $a^{2} c^{2}$ $=(a \cdot b c)^{2}$. If $a$ and $b c$ do not commute, $b c$ has finite order, and so does $c$. Suppose $a \cdot b c=b c \cdot a$. Then (3) becomes

$$
a^{2} c^{2}=a^{2}(b c)^{2}=a^{2}\left(b^{2} c^{2}\right) .
$$

Therefore $b^{2}=1$, a contradiction since $b$ has order 4 .

Theorem V. A Hamiltonian, di-associative loop $L$ is either an Abelian group or the direct product of an Abelian group with elements of odd order and a loop $H$ with the following properties:

(i) The commutator of $H$ consists of the elements of order 1 or 2,

(ii) If $a, b, c, \cdots$ are elements not in the commutator, then $a^{2}=b^{2}$ $=c^{2}=\cdots \neq 1, a^{4}=b^{4}=c^{4}=\cdots=1$.

(iii) If $a, b$ do not commute, $\{a, b\}$ is a quaternion group. (Since $H$ is assumed not Abelian, there exists at least one such pair of elements.) If a, $b$ commute, then $a=t b$ where $t$ is an element of the commutator.

(iv) If $a, b$ do not commute and if $h$ is an element of $H$ which commutes with every element of $\{a, b\}$, then $h$ is an element of the commutator.

Proof. If $L$ is commutative, by Theorem III it is an Abelian group. If it is not commutative, by Theorem IV every element has finite order and so by Theorem II it is the direct product of $p$-loops. By Corollary IIIa, $L$ is then the direct product of a 2-loop $H$ and an Abelian group with elements of odd order.

From the di-associativity, every two elements $a, b$ of $H$ generate a subgroup which is either Abelian or Hamiltonian, and in the latter case it is a quaternion group. ${ }^{4}$ Since $H$ is not Abelian, it contains at least two elements $a, b$ such that $\{a, b\}=Q$ is a quaternion group. If $z$ is an element not in the commutator of $H$, there exists $h \in H$ such that $\{h, z\}$ is a quaternion group, and so $z$ has order 4 .

To show that the commutator consists of the unit element and the elements of order 2, we note first that if $c^{2}=1$, then $a c=d a$ where $d \in\{c\}$ so $d=c$. Conversely suppose that $c$ commutes with every element of $Q$. There exists $d \in\{a\} \cap\{b\} \cap\{c\}$ such that

$$
a^{2} \cdot c^{2}=(a b)^{2} \cdot c^{2}=(a b \cdot c)^{2}=(d[a \cdot b c])^{2}=d^{2}(a \cdot b c)^{2} .
$$

By the same argument as in the proof of Theorem IV, $d^{2}=1$ and this becomes:

\footnotetext{
4 The author wishes to thank the referee for his suggestions which substantially shorten this proof.
} 


$$
a^{2} c^{2}=(a \cdot b c)^{2} \text {. }
$$

If $a$ and $b c$ commute, this further becomes

$$
a^{2} c^{2}=a^{2}(b c)^{2}=a^{2} \cdot b^{2} c^{2} .
$$

So $b^{2}=1$, a contradiction to the choice of $b$. If $a$ and $b c$ do not commute, they generate a quaternion group and $a^{2}=(b c)^{2}=b^{2} c^{2}=a^{2} c^{2}$ since $a^{2}=b^{2}$. Therefore $c^{2}=1$.

We have proved, moreover, part (iv). If $c$ is not an element of the commutator, there exists an element $q \in Q$ which does not commute with $c$ so $\{q, c\}$ is a quaternion group and $q^{2}=c^{2}, q^{4}=c^{4}=1$.

In general, if $x, y$ commute but are not elements of the commutator, since $y^{4}=1$ we have $x=x y^{4}=\left(x y^{3}\right) y$. Let $t=x y^{3}$. Then $t^{2}=\left(x y^{3}\right)^{2}=1$, so $t$ is an element of the commutator.

The complete structure theory of the loop may be given if an additional associativity condition is imposed. Condition A: if three distinct elements of a loop associate in some order, then the subloop generated by the three elements is a group. In particular the class of Moufang loops [2, p. 293] satisfies Condition A; however, there exist examples of loops which are di-associative and satisfy Condition A but which are not Moufang.

If the Latin square given below is considered as the multiplication table of a loop, every two independent elements generate a group of order 4 so it is di-associative. No three independent elements associate in any order so that the loop trivially satisfies Condition A. However, it is not Moufang since $[(3 \cdot 5) \cdot 8] \cdot 5=4$ while $3 \cdot[5 \cdot(8 \cdot 5)]$ $=7$. The loop is

$\begin{array}{rrrrrrrrrr}1 & 2 & 3 & 4 & 5 & 6 & 7 & 8 & 9 & 10 \\ 2 & 1 & 4 & 3 & 8 & 9 & 10 & 5 & 6 & 7 \\ 3 & 4 & 1 & 2 & 9 & 10 & 8 & 7 & 5 & 6 \\ 4 & 3 & 2 & 1 & 10 & 8 & 9 & 6 & 7 & 5 \\ 5 & 8 & 9 & 10 & 1 & 7 & 6 & 2 & 3 & 4 \\ 6 & 9 & 10 & 8 & 7 & 1 & 5 & 4 & 2 & 3 \\ 7 & 10 & 8 & 9 & 6 & 5 & 1 & 3 & 4 & 2 \\ 8 & 5 & 7 & 6 & 2 & 4 & 3 & 1 & 10 & 9 \\ 9 & 6 & 5 & 7 & 3 & 2 & 4 & 10 & 1 & 8 \\ 10 & 7 & 6 & 5 & 4 & 3 & 2 & 9 & 8 & 1 .\end{array}$

The basis elements of the Cayley-Dickson algebra [3, p. 561] form a loop with three generators $a_{1}, a_{2}, a_{3}$ which satisfy the relationships: 


$$
\begin{aligned}
& a_{i}^{4}=1 ; \quad a_{i}^{2}=a_{j}^{2} ; \quad a_{i} a_{j}=a_{j}^{8} a_{i} ; \quad i \neq j ; \\
& a_{i} a_{i} \cdot a_{k}=\stackrel{3}{a_{i}} \cdot a_{j} a_{k} ; \quad i, j, k \text { all different. }
\end{aligned}
$$

This loop may be conveniently called the Cayley loop.

Theorem VI. A Hamiltonian, di-associative loop satisfying Condition A is (i) an Abelian group, or (ii) a Hamiltonian group, or (iii) the direct product of a Cayley loop, an Abelian group with exponent 2, and an Abelian group with elements of odd order.

Proof. Applying Theorem V we have left only to show that the loop $H$ there defined is the direct product of a Cayley loop and an Abelian group with exponent 2 .

Let the elements $a$ and $b$ of $H$ generate a quaternion group $Q$, and let $K$ be the set of all elements of $H$ not in $Q$ which commute with at least one element of order 4 of $Q$. If $k$ is an element of order 4 of $K$, suppose that $a k=k a$. (If $k$ commutes with some other element of order 4 of $Q$, the proof is the same.) Then $\{a, k\}=\left\{a, k_{1}\right\}$ where $k=a k_{1}$, so $k_{1}=a^{3} k$. By Theorem $\mathrm{V}(\mathrm{i})$ and $\mathrm{V}$ (ii), $k^{2}=a^{2}$ and we have $k_{1}^{2}=a^{2} k^{2}=1$, since $a$ and $k$ are commutative. Let $K_{1}$ consist of the unit element and all elements of $H$ of order 2 except $a^{2}$. Then $K_{1}$ is clearly a loop, moreover an Abelian group with exponent 2. Also $k_{1}$ is an element of $K_{1}$, so $k$, which equals $a k_{1}$, is an element of $Q \times K_{1}$. Therefore $\{Q, K\} \subset Q \times K_{1}$. Conversely since $K \supset K_{1}$, we have $\{Q, K\}$ $\supset Q \times K_{1}$. Combining these we have $\{Q, K\}=Q \times K_{1}$.

Let $L$ be the set of elements of $H$ not in $Q \times K_{1}$. Then an element of $L$ does not commute with any element of order 4 of $Q$. If an element $s$ of $L$ associates in any order with two independent elements of $Q$-suppose they are $a$ and $b$-then $\{a, b, s\}$ is a group by Condition $\mathrm{A}$, and by the theory of Hamiltonian groups $\{a, b, s\}=Q \times\left\{s_{1}\right\}$ where $s_{1}^{2}=1$. Then $s=y s_{1}$ for some element $y$ of order 4 of $Q$. So

$$
y s=y \cdot y s_{1}=y s_{1} \cdot y=s y
$$

and $s$ commutes with $y$, in contradiction to the choice of $L$. Therefore $\{a, b, s\}$ is not a group for any $s$ in $L$.

Let $c$ be any element of $L$. That $C=\{a, b, c\}$ is a Cayley loop may be easily seen by Theorem V and the preceding remarks. Let $\bar{K}$ be the set of elements which commute with at least one element of order 4 of $C$. As before $\{C, \bar{K}\}=C \times K_{1}$ with $K_{1}$ defined as above. Also as before if $\bar{L}$ is the set of elements of $H$ not in $C \times K_{1}$, then $\{x, y, \bar{s}\}$ is not a group for any pair of independent elements $x, y$ of $C$ and any element $\bar{s}$ of $\bar{L}$. We shall show that $\bar{L}$ is an empty set.

Let $d$ be any element of $\bar{L}$. Then 


$$
a b \cdot c d=\left(a^{3} b \cdot c\right) d=(a \cdot b c) d=a^{3}(b c \cdot d)=a(b \cdot c d),
$$

so $\{a, b, c d\}$ is a group by Condition A. By the theory of Hamiltonian groups, $\{a, b, c d\}=\{a, b\} \times\{t\}$ where $t^{2}=1$. Therefore $c d=q t$ for some $q$ an element of $\{a, b\}$, and $d=c^{3} q \cdot t$; so $d$ is an element of $C \times K_{1}$ contrary to its choice. The set $\bar{L}$ must be empty and $H=C \times K_{1}$.

Theorem VII (Converse of Theorem V). If $A$ is an Abelian group with elements of odd order, if $T$ is an Abelian group with exponent 2, and if $K$ is a di-associative loop with the following properties:

(i) The elements of $K$ have order 1, 2, or 4,

(ii) $K$ contains at least two elements $a$ and $b$ such that $\{a, b\}$ is $a$ quaternion group,

(iii) Every element of order 2 of $K$ is in the center,

(iv) If $x, y, z$ are elements of order 4 of $K$, then $x^{2}=y^{2}=z^{2}, x y=d \cdot y x$ where $d=1$ or $x^{2}$, and $x y \cdot z=e(x \cdot y z)$ where $e=1$ or $x^{2}$;

then their direct product $A \times T \times K$ is a di-associative Hamiltonian loop.

Proof. It is clear from Theorem I that if $T \times K$ is Hamiltonian, then $A \times T \times K$ is Hamiltonian since the order of an element of $A$ is odd while the order of an element of $T \times K$ is $2^{n}$.

To prove that $K$ is Hamiltonian we note that if $\mathfrak{B}$ is any permutation of the inner mapping group of $K$ and $k \in K$, then $k \mathfrak{B}=t k$ where $t=1$ or $x^{2}$. For instance, if $\mathfrak{B}=R_{x, y}$, then

$$
k \Re=k R_{x} R_{y} R_{x y}^{-1}=(k x \cdot y) R_{x y}^{-1}=(t k \cdot x y) R_{x y}^{-1}=t k
$$

where $t=1$ or $x^{2}$. If $k$ has order 2 , since it is central, $t=1$ and $k \mathfrak{B}=k$. Let $K^{\prime}$ be any subloop of $K$. If $K^{\prime}$ contains an element $k$ of order 4, then $k^{2}=x^{2}$, so for any $k^{\prime} \in K^{\prime}$, then $K^{\prime}$ contains $x^{2} k^{\prime}$. Thus $K^{\prime}$ is invariant under any transformation of the inner mapping group. If $K^{\prime}$ contains no element of order 4 , its elements are all central and $K^{\prime}$ is invariant.

To prove that $T \times K$ is Hamiltonian, suppose that $K^{\prime}$ and $\bar{K}^{\prime}$ are subloops of $K$ and that $T^{\prime}$ and $\bar{T}^{\prime}$ are subloops of $T$ such that $K^{\prime} / \bar{K}^{\prime} \cong T^{\prime} / \bar{T}^{\prime}$. The loop $T^{\prime} / \bar{T}^{\prime}$ is Abelian with exponent 2 and therefore so is $K^{\prime} / \bar{K}^{\prime}$. Every element of $K^{\prime} / \bar{K}^{\prime}$ has order 2 and so it is in the center of $K / \bar{K}^{\prime}$. By Theorem I, $A \times K$ is Hamiltonian.

Corollary VIIa (Converse of Theorem VI). The direct product of an Abelian group with elements of odd order, an Abelian group with exponent 2, and a Cayley loop is a di-associative Hamiltonian loop satisfying Condition A. 


\section{REFERENCES}

1. A. A. Albert, Quasigroups. I, Trans. Amer. Math. Soc. vol. 54 (1943) pp. 507519.

2. R. H. Bruck, Contributions to the theory of loops, Trans. Amer. Math. Soc. vol. 60 (1946) pp. 245-354.

3. H. S. M. Coxeter, Integral Cayley numbers, Duke Math. J. vol. 13 (1946) pp. 561-577.

4. H. Zassenhaus, The theory of groups, New York, Chelsea, 1949.

UNIVERSITY OF WISCONSIN 